\title{
Diagnostic accuracy of fine-needle aspiration of solid pancreatic lesions guided by endoscopic ultrasound elastography
}

\author{
Antonio Facciorusso, Matteo Martina, Rosario Vincenzo Buccino, Maurizio Cosimo Nacchiero, \\ Nicola Muscatiello
}

University of Foggia, Italy

\section{Abstract}

\begin{abstract}
Background Real-time elastography (RTE) may increase the diagnostic accuracy of fine-needle aspiration guided by endoscopic ultrasound. The aim of this study was to establish the diagnostic accuracy, sensitivity, and specificity of this combined methodological approach in a cohort of patients with solid pancreatic masses.
\end{abstract}

Methods We reviewed data from 54 patients with solid pancreatic lesions referred to our institution between January 2014 and June 2015. RTE, assessed in terms of strain ratio, was performed both qualitatively and semi-quantitatively, and a $25 \mathrm{G}$ needle was inserted into the most suspicious part of the lesion. Sensitivity, specificity, diagnostic accuracy, positive and negative predictive values were calculated.

Results The median lesion size was $35 \mathrm{~mm}$ (interquartile range: $25-43 \mathrm{~mm}$ ). A diagnosis of adenocarcinoma was confirmed in $85.1 \%$ of cases. RTE, with a strain ratio cutoff of 4.21 , showed a sensitivity of $86.9 \%$, a specificity of $75 \%$, and diagnostic accuracy of $85.1 \%$. The diagnostic accuracy, sensitivity, and specificity of the combined methodology were $94.4 \%, 93.4 \%$, and $100 \%$, respectively. The positive predictive value was $100 \%$, the negative predictive value $72.7 \%$ and the negative likelihood ratio 6.5. No severe adverse events were registered.

Conclusion The combination of RTE with endoscopic ultrasound-guided fine-needle aspiration appears to be an efficient and safe technique for the characterization of solid pancreatic masses.

Keywords Real-time elastography, endoscopic ultrasound, pancreatic cancer, sensitivity

Ann Gastroenterol 2018; 31 (4): 1-6

\section{Introduction}

Diagnostic assessment of solid pancreatic lesions may represent a real challenge in clinical practice, even with the aid of tissue sampling by means of fine-needle aspiration (FNA) guided by endoscopic ultrasound (EUS).

EUS-guided FNA, although showing an excellent specificity up to $100 \%$, has a variable sensitivity (ranging from $85 \%$ to over 93\%) that depends on several factors, such as the availability of rapid on-site tissue evaluation (ROSE) by a cytopathologist, or the needle caliber [1-4]. This variability may lead to false-

Gastroenterology Unit, University of Foggia, Italy

\section{Conflict of Interest: None}

Correspondence to: Antonio Facciorusso, Department of Medical Sciences, Gastroenterology Unit, University of Foggia, Viale L. Pinto, 1, 71100 Foggia, Italy, e-mail: antonio.facciorusso@virgilio.it

Received 23 January 2018; accepted 20 March 2018; published online 7 May 2018

DOI: https://doi.org/10.20524/aog.2018.0271 negative results, particularly in cases of pancreatic cancer occurring in patients with chronic pancreatitis.

In order to increase the accuracy of EUS for the evaluation of pancreatic masses, specific devices already used in other fields (for instance in liver oncology) have been developed, including contrast-enhanced harmonic (CEH) EUS [5] and real-time elastography (RTE) EUS [6,7]. The interesting performance of these two techniques (CEH-EUS and RTE-EUS, either isolated or combined) in terms of negative predictive value has raised hopes of improving the diagnostic algorithm for pancreatic masses, particularly in those difficult cases when EUS-FNA results are inconclusive $[7,8]$.

However, none of these studies tested whether EUS elastography could be helpful in guiding tissue sampling and hence increasing the diagnostic yield of EUS-FNA. Elastography-guided biopsies have already been used for a long time in urology, where they have been shown to increase the detection rate of prostate cancer $[9,10]$. In fact, the difference in tissue elasticity between malignant and benign lesions and among different parts within the same solid mass could be exploited to correctly and precisely target the "harder" part of the lesion (more likely to be malignant), thus decreasing the 
risk of negative or non-diagnostic samples. Based on these principles, the aim of our study was to establish the diagnostic accuracy, sensitivity, and specificity of RTE-guided EUS-FNA in a retrospective series of patients with solid pancreatic masses.

\section{Patients and methods}

\section{Patients}

From a prospectively collected database, we reviewed data for 54 consecutive patients out of 83 subjects with solid pancreatic lesions, referred to our institution between January 2014 and June 2015. All patients had been diagnosed with pancreatic masses by means of transabdominal ultrasonography, computed tomography scan or magnetic resonance imaging. The Institutional Review Board approved this retrospective report and written informed consent was obtained from all the patients.

The following exclusion criteria were used: age under 18 years, cystic pancreatic lesions, lesions $<1 \mathrm{~cm}$, history of previous gastrectomy, severe coagulopathy or current anticoagulant/antiaggregant therapy that could not be suspended, inability or refusal to provide informed consent. All procedures were performed by a board-certified gastroenterologist (NM), who had performed more than 600 EUS-FNA and 150 RTE-EUS before the study period.

\section{Technical procedure}

The technique used for diagnostic and interventional EUS at our center has been described elsewhere [11,12]. Briefly, with the patient under sedation with propofol, EUS was conducted using a Pentax FG-36UA ultrasound endoscope (Pentax Europe, Ltd., Hamburg, Germany) with a curved-array transducer. After introduction to the stomach, the endoscopic ultrasound probe was placed in contact with the gastric wall and the aorta was identified in an elongated cross-section-a finding confirmed by color Doppler imaging. The scope was then slowly advanced up to the duodenum when necessary. A 25G needle (EchoTip Ultra, Cook Medical LLC, Bloomington, Indiana, USA), with a central stylet to protect the needle's aspiration channel, was introduced through the endoscope's working channel. A transduodenal approach was used for lesions of the pancreatic head and uncinate process, whereas the trans-gastric approach was used for lesions of the body or tail.

RTE assessment of pancreatic masses was performed using a Hitachi Hi Vision Ascendus device (Hitachi medical systems, Tokyo, Japan). All areas that appeared suspicious on elastography (i.e., those appearing in a dark blue color as a consequence of the higher cellularity of tumoral tissue) were recorded and stored in our database. Both a qualitative assessment, based on a red-green-blue color map, and a semiquantitative approach that provided a numeric value expressed as strain ratio [13] were undertaken.
The needle was inserted into the most suspicious part ("dark blue") of the lesion and immediately after the procedure the stylet was removed. More than 10 to-and-fro movements were made within the lesion and aspiration was obtained with a $10 \mathrm{~cm}^{3}$ suction syringe applied to the hub of the FNA device. Two passes were performed in most patients. At the end of the procedure, the needle was retracted and the samples were fixed in $95 \%$ ethanol solution. After being grossly checked for adequacy, samples were prepared for cytological examination with Papanicolaou staining. Patients were continuously monitored during the procedure by a board-certified anesthesiologist with an automated noninvasive blood pressure device, electrocardiogram tracing and pulse oximetry. The procedure adopted in the current study is depicted in Fig. 1.

\section{Cytology}

The degree of cellularity and blood contamination of specimens was graded as five levels (0: none; 1 : minimal; 2 : mild; 3: moderate; 4: many) and cellularity was then divided into two groups: "high cellularity" indicated a cellular level of 3 or 4 and "low cellularity" indicated a level of 0,1 or 2 [14].

The reference standard for classification was surgery or death from pancreatic cancer in those subjects unsuitable for surgery. In particular, if after a follow up of 12-18 months there was no sign of disease progression, or if disease regression was registered, the lesion was classified as inflammatory [15].

Lesions diagnosed as malignant by cytopathology of the EUS-FNA sample and were finally confirmed by surgery or clinical course were considered to be true positives; likewise, benign aspirates finally diagnosed as benign were considered to be true negatives. On the other hand, aspirates, apparently benign on cytopathological examination but finally diagnosed as malignant, were considered to be false negatives. Nondiagnostic or inconclusive samples were registered as such in the database and for analytical purposes were classified as false negatives when diagnostic accuracy was computed.

\section{Follow up}

All clinical and safety outcomes, as well as imaging follow up, were assessed by clinicians blinded to the EUS technique adopted. Adverse event rates were evaluated during the procedure, before discharge, and at 1 and 3 days by means of telephone calls. After the first month, all the clinical visits, which included physical examination, laboratory analyses (including tumor markers) and imaging evaluation, were performed on an outpatient basis at 50 days after the procedure and every 3 months thereafter.

\section{Statistical analysis}

Categorical variables were expressed as frequencies and percentages and continuous variables as medians and 


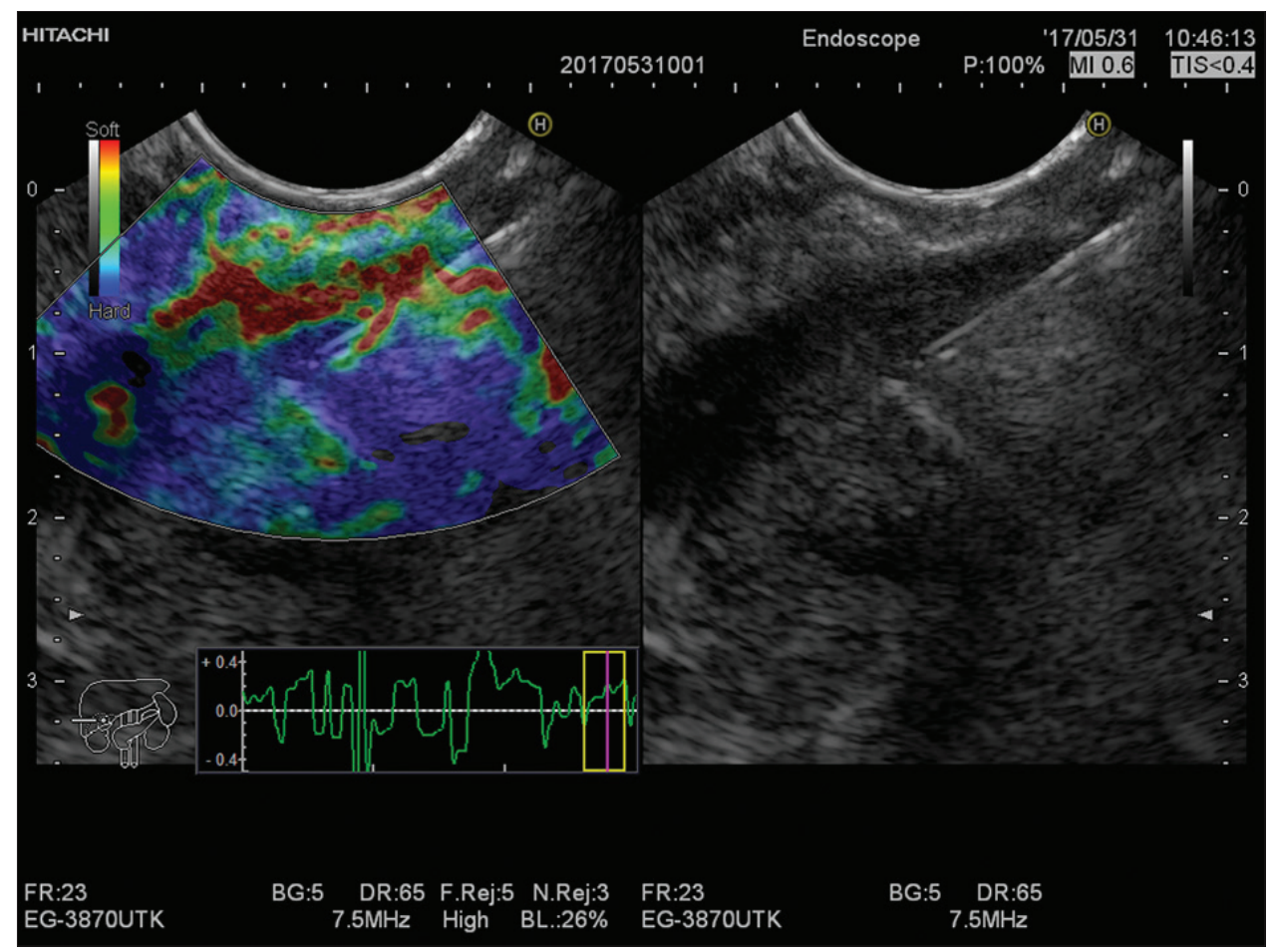

Figure 1 An endoscopic ultrasonography image describing real-time elastography-targeted fine-needle aspiration of a solid lesion located in the pancreatic head. The needle was inserted into the most suspicious part ("dark blue") of the lesion. The final diagnosis identified ductal adenocarcinoma

interquartile ranges (IQRs). Sensitivity, specificity, diagnostic accuracy, positive and negative predictive values were calculated. Sensitivity was computed as the proportion of positives correctly identified by the test among the prevalence of malignancy in the study cohort. Specificity was calculated as the proportion of negatives correctly identified as such among the patients not affected by the tumor in the study cohort. Positive likelihood ratio was defined as sensitivity/falsepositive rate and negative likelihood ratio as false-negative rate/specificity. The analysis was performed using $\mathrm{R}$ Statistical Software (Foundation for Statistical Computing, Vienna, Austria) and significance was established at the 0.05 level (twosided).

\section{Results}

\section{Patients}

Table 1 summarizes the main characteristics of the study population. The median age was 68 years (IQR: $59-73$ ) and $64.8 \%$ of patients were male. The majority of lesions were located in the pancreatic head or uncinate $(62.9 \%)$ and the median size was $35 \mathrm{~mm}$ (IQR: 25-43). About one fifth of the whole population (20.3\%) finally underwent surgery and the diagnosis of adenocarcinoma was confirmed from a surgical specimen or by the patient's clinical course in $85.1 \%$ of cases.
Table 1 Baseline characteristics of the study population

\begin{tabular}{lc}
\hline Variable & Total (54 patients) \\
\hline Age (years) & $68(59-73)$ \\
Sex (male) & $35(64.8 \%)$ \\
\hline $\begin{array}{l}\text { Location } \\
\text { Head/Uncinate } \\
\text { Body/Tail }\end{array}$ & $34(62.9 \%)$ \\
Size (mm) & $20(37.1 \%)$ \\
Surgery & $35(25-43)$ \\
Final diagnosis (adenocarcinoma) & $11(20.3 \%)$ \\
\hline Number of needle passes per lesion & $46(85.1 \%)$ \\
\hline $\begin{array}{l}\text { Variables are expressed as median (interquartile range) or absolute number } \\
\text { (percentage) where appropriate. } \\
\text { *Expressed as mean } \pm \text { standard deviation }\end{array}$ & $2 \pm 1.1$ \\
\hline
\end{tabular}

\section{RTE findings}

The quantitative elastography score was represented by the strain ratio method, i.e., as the ratio between a surrounding non-mass area (area B) and the mass or region of interest (area A) [13]. Based on the results of the receiver operating characteristic curve used for analysis (Fig. 2), the cutoff level of strain ratio able to achieve the maximal accuracy (expressed as area under the curve) was 4.21 , which provided a sensitivity of $86.9 \%$, a specificity of $75 \%$, and a diagnostic accuracy of $85.1 \%$ (Table 2). When the cutoff value of 6.04 (proposed in 


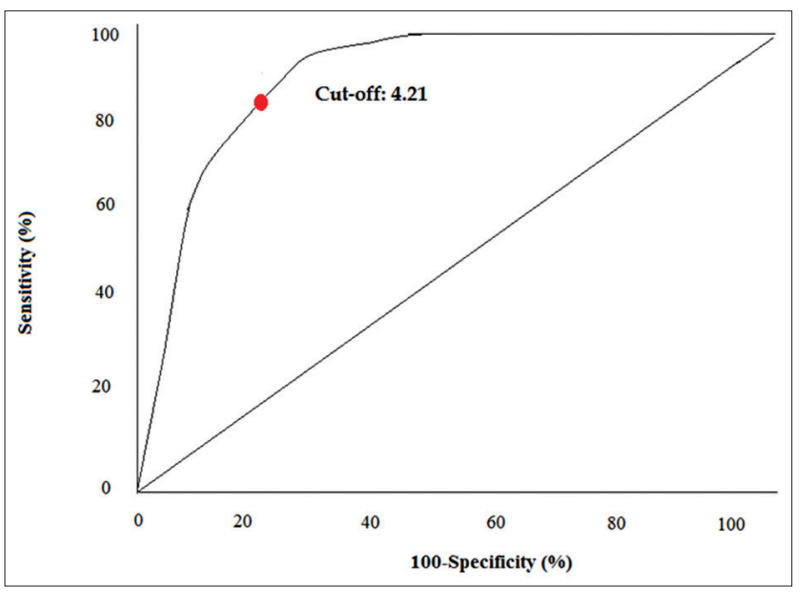

Figure 2 Receiver operating characteristic (ROC) curve analysis aimed at identifying the best cutoff point for elastography strain ratio. ROC curve analysis identified the value 4.21 as the most sensitive and specific cutoff point for the elastography strain ratio

the landmark study by Iglesias-Garcia et al [13]) was adopted, specificity increased significantly to $87.5 \%$ but sensitivity dropped to $80.4 \%$, resulting in a slight decrease in overall accuracy (81.4\%) (Table 2). Pancreatic ductal adenocarcinoma appeared as homogeneous hard (blue) tissue in $20 \%$ of cases, while we found areas with different strain (mainly due to necrosis) that allowed targeting the hardest area specifically in $80 \%$ of lesions.

\section{Diagnostic accuracy of RTE-targeted EUS-FNA}

All diagnostic outcomes are reported in Table 3. No needle malfunctions nor mechanical failures occurred and technical success was $100 \%$. A cytological diagnosis positive for malignancy was found in 43 cases $(79.6 \%)$, while 10 samples were negative (18.6\%) and in one single case the cytological sample was rated as inadequate (1.8\%).

Diagnostic accuracy, sensitivity, and specificity were $94.4 \%$, $93.4 \%$ and $100 \%$, respectively. Since no false positives were observed, positive predictive value was $100 \%$ and positive likelihood ratio could not be computed (false-positive rate, the denominator of the proportion, was 0 ). On the other hand, 3 false negatives were registered; hence, the negative predictive value was $72.7 \%$ and the negative likelihood ratio was 6.5 (Table 3).

\section{Cytological findings and adverse events}

According to commonly accepted scores published elsewhere $[14,15]$, overall cellularity was rated as average (2.5, IQR: 1-4), as were blood contamination (2, 0-3) and neoplastic cellularity $(2,1-4)$. Contamination by cellular debris or amorphous material did not represent a real issue, since a low rate for this specific item was given by the cytopathologist $(1,0-2)$ (Table 4).
Table 2 Elastography findings

\begin{tabular}{lcc}
\hline Diagnostic outcome & $\begin{array}{c}\text { Strain ratio } \\
4.21^{\mathrm{a}}\end{array}$ & $\begin{array}{c}\text { Strain ratio } \\
6.04^{\mathrm{b}}\end{array}$ \\
\hline Positive for malignancy & $42(77.7 \%)$ & $38(70.3 \%)$ \\
\hline Negative for malignancy & $12(22.3 \%)$ & $16(29.7 \%)$ \\
\hline Accuracy & $46 / 54(85.1 \%)$ & $44 / 54(81.4 \%)$ \\
\hline Sensitivity & $40 / 46(86.9 \%)$ & $37 / 46(80.4 \%)$ \\
\hline Specificity & $6 / 8(75 \%)$ & $7 / 8(87.5 \%)$ \\
\hline Positive predictive value & $40 / 42(95.2 \%)$ & $37 / 38(97.4 \%)$ \\
\hline Negative predictive value & $6 / 12(50 \%)$ & $7 / 16(43.7 \%)$ \\
\hline Positive likelihood ratio & 3.48 & 6.43 \\
Negative likelihood ratio & 0.17 & 0.22 \\
\hline Variables are expressed as proportion (percentage) \\
${ }^{\mathrm{a} C u t o f f}$ value identified using the receiver operating characteristic curve \\
${ }^{\mathrm{b} C u t o f f}$ value identified in previous studies ${ }^{13}$ &
\end{tabular}

Table 3 Diagnostic performance of the procedure

\begin{tabular}{lc}
\hline Diagnostic outcome & Total (54 patients) \\
\hline Positive for malignancy & $43(79.6 \%)$ \\
Negative for malignancy & $10(18.6 \%)$ \\
Sample inadequacy & $1(1.8 \%)$ \\
\hline Accuracy & $51 / 54(94.4 \%)$ \\
\hline Sensitivity & $43 / 46(93.4 \%)$ \\
\hline Specificity & $8 / 8(100 \%)$ \\
Positive predictive value & $43 / 43(100 \%)$ \\
Negative predictive value & $8 / 11(72.7 \%)$ \\
\hline Positive likelihood ratio & $\mathrm{NA}$ \\
Negative likelihood ratio & 6.5 \\
\hline
\end{tabular}

Variables are expressed as proportion (percentage)

NA, not assessable

Table 4 Cytological findings

\begin{tabular}{lc}
\hline Variable & Score \\
\hline Overall cellularity & $2.5(1-4)$ \\
Blood contamination & $2(0-3)$ \\
$\begin{array}{l}\text { Presence of cellular } \\
\text { debris }\end{array}$ & $1(0-2)$ \\
Neoplastic cellularity & $2(1-94)$ \\
\hline Variables are expressed as median (interquartile range) &
\end{tabular}

No severe adverse events were registered. Five patients experienced a slight increase in pancreatic enzyme levels (9.2\%) and 3 complained about mild abdominal pain (5.5\%). Mild post-procedural bleeding was observed in 2 cases $(3.7 \%)$. All of these complications were managed conservatively. 


\section{Discussion}

EUS-FNA represents a very useful diagnostic tool for characterizing solid pancreatic lesions, as confirmed in several studies published in the last decade. The great strength of FNA is its excellent specificity (around 100\% in nearly all studies), while there are still conflicting results regarding its sensitivity (which ranges from $85-93 \%$ in most trials) and sample adequacy $[1,2]$. Many technical aspects, such as needle caliber or the availability of ROSE [1-4], and several devices have been investigated and developed in order to improve the diagnostic performance of EUS-FNA, but none of them was proven determinant as regards reaching an optimal diagnostic accuracy.

RTE provides additional information about lesion characteristics and it has been used for long time in other fields of human oncology [16]. Tumors are usually harder than normal surrounding tissues and this peculiar feature can be used to achieve a more careful characterization of pancreatic lesions through EUS [16]. Nevertheless, RTE-EUS alone shows relatively low specificity (usually below $80 \%$ ) [13,17]; hence, there is a need to adopt complementary diagnostic tools such as CEH-EUS [7,8] or standard EUS-FNA [18].

To the best of our knowledge, our study represents the first report of RTE-EUS-targeted FNA, a technique already in use for prostate $[9,10]$ and thyroid cancer [19], in pancreatic solid lesions. The RTE-EUS findings observed in our series are comparable to those published elsewhere $[13,18]$, although we adopted a different cutoff for strain ratio (4.21). RTE-EUS alone was confirmed to have good sensitivity (86.9\%) and specificity (75\%), thus resulting in $85.1 \%$ accuracy (Table 2). In order to increase the standardization of our results, we performed an additional analysis adopting the cutoff value of 6.04 (proposed in the landmark study by Iglesias-Garcia et al [13]), as a result of which the overall accuracy slightly decreased to $81.4 \%$.

RTE-EUS-guided FNA proved effective in providing adequate samples (only 1 case of inadequacy) and the diagnostic outcomes were similar to those of other studies conducted with the aid of ROSE or a superior number of needle passes $[14,15,19-23]$. In the present study, diagnostic accuracy, sensitivity, and specificity were $94.4 \%, 93.4 \%$, and $100 \%$, respectively (Table 3). RTE-EUS-targeted FNA confirmed the excellent positive predictive value of FNA (100\%) and showed an encouraging negative predictive value of $72.7 \%$, with a negative likelihood ratio of 6.5 (Table 3 ). These valuable results can be interpreted as a consequence of the adequacy of the samples collected, as demonstrated by the optimal scores for overall and neoplastic cellularity (median 2.5 and 2, respectively) and blood contamination (median 2; Table 4).

We can argue that RTE-EUS guidance is able to avoid necrotic areas or zones with low cellularity, an aspect which always represented an issue limiting the adequacy of the sample and therefore the diagnostic performance of conventional EUSFNA. RTE-EUS is less time-consuming and safer than CEHEUS; hence, our results may pave the way to a broader use of this technique (at least as a complement to FNA) in pancreatic endoscopic ultrasound. Finally, as expected, no severe adverse events were observed in our series, confirming the excellent safety profile of RTE-EUS and of EUS-FNA.

This study has a number of strengths: first of all, it is the first report on the diagnostic efficacy of RTE-EUS-targeted FNA for the detection of pancreatic malignancies. Second, the completeness of the data collected strengthens the results of our analysis. Third, the fact that it was a single-center study eliminates the possibility of bias due to different technical procedures or endoscopic training.

Nevertheless, the paper has a main limitation in the form of its retrospective nature, which could have led to selection or outcome report biases. However, the adequacy of follow up and the completeness of the recorded data support the reliability of our findings. Moreover, most reported outcomes were objective parameters, and thus not amenable to reporting alterations. Other limitations were the relatively small number of patients recruited, the lack of data on lesions other than adenocarcinoma or chronic pancreatitis (such as neuroendocrine tumors), and the absence of a control cohort undergoing traditional EUS-FNA.

Despite such limitations, our analysis provides robust evidence in favor of a novel, safe and effective echoendoscopic diagnostic technique based on RTE-EUS-targeted FNA of solid pancreatic lesions, with encouraging sensitivity and specificity. Prospective randomized trials are warranted to confirm the results of our analysis.

\section{Summary Box}

\section{What is already known:}

- Endoscopic ultrasound (EUS)-guided fine-needle aspiration (FNA), although showing excellent specificity, has variable sensitivity

- To increase the accuracy of EUS in the evaluation of pancreatic masses, specific techniques, such as contrast-enhanced harmonic EUS and real-time elastography (RTE) EUS, have been developed

\section{What the new findings are:}

- RTE-EUS-guided FNA appears to be an efficient and safe technique with encouraging sensitivity and specificity in the characterization of solid pancreatic masses

- Prospective randomized trials are warranted to confirm the results of our analysis

\section{References}

1. Jani BS, Rzouq F, Saligram S, et al. Endoscopic ultrasound-guided fine-needle aspiration of pancreatic lesions: a systematic review of technical and procedural variables. N Am J Med Sci 2016;8:1-11.

2. Matsubayashi H, Matsui $T$, Yabuuchi $Y$, et al. Endoscopic 
ultrasonography guided-fine needle aspiration for the diagnosis of solid pancreaticobiliary lesions: clinical aspects to improve the diagnosis. World J Gastroenterol 2016;22:628-640.

3. Iglesias-Garcia J, Lariño-Noia J, Abdulkader I, Domínguez-Muñoz JE. Rapid on-site evaluation of endoscopicultrasound-guided fine-needle aspiration diagnosis of pancreatic masses. World J Gastroenterol 2014;20:9451-9457.

4. Facciorusso A, Stasi E, Di Maso M, Serviddio G, Ali Hussein MS, Muscatiello N. Endoscopic ultrasound-guided fine needle aspiration of pancreatic lesions with 22 versus 25 Gauge needles: a meta-analysis. United European Gastroenterol J 2017;5:846-853.

5. Gong TT, Hu DM, Zhu Q. Contrast-enhanced EUS for differential diagnosis of pancreatic mass lesions: a meta-analysis. Gastrointest Endosc 2012;76:301-309.

6. Dietrich CF, Săftoiu A, Jenssen C. Real time elastography endoscopic ultrasound (RTE-EUS), a comprehensive review. Eur J Radiol 2014;83:405-414.

7. Săftoiu A, Iordache SA, Gheonea DI, et al. Combined contrastenhanced power Doppler and real-time sonoelastography performed during EUS, used in the differential diagnosis of focal pancreatic masses (with videos). Gastrointest Endosc 2010;72:739-747.

8. Iordache S, Costache MI, Popescu CF, Streba CT, Cazacu S, Săftoiu A. Clinical impact of EUS elastography followed by contrast-enhanced EUS in patients with focal pancreatic masses and negative EUS-guided FNA. Med Ultrason 2016;18:18-24.

9. Salomon G, Drews N, Autier P, et al. Incremental detection rate of prostate cancer by real-time elastography targeted biopsies in combination with a conventional 10-core biopsy in 1024 consecutive patients. BJU Int 2014;113:548-553.

10. Wang R, Chen JJ, Hu B. Transrectal real-time elastography-guided transperineal prostate biopsy as an improved tool for prostate cancer diagnosis. Int J Clin Exp Med 2015;8:6522-6529.

11. Facciorusso A, Maso MD, Barone M, Muscatiello N. Echoendoscopic ethanol ablation of tumor combined to celiac plexus neurolysis improved pain control in a patient with pancreatic adenocarcinoma. Endosc Ultrasound 2015;4:342-344.

12. Facciorusso A, Di Maso M, Serviddio G, Larghi A, Costamagna G, Muscatiello N. Echoendoscopic ethanol ablation of tumor combined with celiac plexus neurolysis in patients with pancreatic adenocarcinoma. J Gastroenterol Hepatol 2017;32:439-445.

13. Iglesias-Garcia J, Larino-Noia J, Abdulkader I, Forteza J, Dominguez-Munoz JE. Quantitative endoscopic ultrasound elastography: an accurate method for the differentiation of solid pancreatic masses. Gastroenterology 2010;139:1172-1180.

14. Lee JK, Lee KT, Choi ER, et al. A prospective, randomized trial comparing 25-gauge and 22-gauge needles for endoscopic ultrasound-guided fine needle aspiration of pancreatic masses. Scand J Gastroenterol 2013;48:752-757.

15. Fabbri C, Polifemo AM, Luigiano C, et al. Endoscopic ultrasoundguided fine needle aspiration with 22- and 25-gauge needles in solid pancreatic masses: a prospective comparative study with randomisation of needle sequence. Dig Liv Dis 2011;43:647-652.

16. Săftoiu A, Vilmann P, Gorunescu F, et al; European EUS Elastography Multicentric Study Group. Efficacy of an artificial neural network-based approach to endoscopic ultrasound elastography in diagnosis of focal pancreatic masses. Clin Gastroenterol Hepatol 2012;10:84-90.

17. Hirche TO, Ignee A, Barreiros AP, et al. Indications and limitations of endoscopic ultrasound elastography for evaluation of focal pancreatic lesions. Endoscopy 2008;40:910-917.

18. Kongkam P, Lakananurak N, Navicharern P, et al. Combination of EUS-FNA and elastography (strain ratio) to exclude malignant solid pancreatic lesions: A prospective single-blinded study. J Gastroenterol Hepatol 2015;30:1683-1689.

19. Yildirim D, Gurses B, Gurpinar B, et al. The value of ultrasound elastography-guided fine-needle aspiration biopsy of thyroid nodules in reducing nondiagnostic results. J Med Ultrason (2001) 2013;40:169-174.

20. Camellini L, Carlinfante G, Azzolini F, et al. A randomized clinical trial comparing $22 \mathrm{G}$ and $25 \mathrm{G}$ needles in endoscopic ultrasoundguided fine-needle aspiration of solid lesions. Endoscopy 2011;43:709-715.

21. Vilmann P, Săftoiu A, Hollerbach S, et al. Multicenter randomized controlled trial comparing the performance of 22 gauge versus 25 gauge EUS-FNA needles in solid masses. Scand J Gastroenterol 2013;48:877-883.

22. Gimeno-García AZ, Elwassief A, Paquin SC, Gariépy G, Sahai AV. Randomized controlled trial comparing stylet-free endoscopic ultrasound-guided fine-needle aspiration with 22-G and 25-G needles. Dig Endosc 2014;26:467-473.

23. Carrara S, Anderloni A, Jovani M, et al. A prospective randomized study comparing $25-\mathrm{G}$ and $22-\mathrm{G}$ needles of a new platform for endoscopic ultrasound-guided fine needle aspiration of solid masses. Dig Liver Dis 2016;48:49-54. 